\title{
Towards Self-Control of Service Rate for Battery Management in Energy Harvesting Devices
}

\author{
Elvina Gindullina and Leonardo Badia \\ University of Padova, Dept. of Information Engineering, via Gradenigo 6B, 35131 Padova, Italy \\ email: \{elvina.gindullina, badia\}@ dei.unipd.it
}

\begin{abstract}
We consider the operation of an energy harvesting wireless device (sensor node) powered by a rechargeable battery, taking non-idealities into account. In particular, we consider sudden decrease and increase of the battery level (leakage and charge recovery consequently) due to the inner diffusion processes in the battery. These processes are affecting the stability of the device operation. In particular, leakage accelerates the depletion of the battery, which results in inactive periods of the device and, thus, potential data loss.

In this paper, we propose a simplified self-control management of a battery expressed by restrictions, which could be used for an efficient operational strategy of the device. To achieve this, we rely on the double-queue model which includes the imperfections of the battery operation and bi-dimensional battery value. This includes both apparent, i.e., available at the electrodes and true energy levels of a battery. These levels can be significantly different because of deep discharge events and can equalize thanks to charge recovery effect. We performed some simulation and observed that we can diminish the models variable number to predict possible unwanted events such as apparent discharge events (when the areas near electrodes are depleted while other areas of the battery still contain some energy) and data losses. This observation helps to achieve sufficiently effective self-control management by knowing and managing just few parameters, and therefore offers valuable directions for the development of autonomic and self-sustainable operation.
\end{abstract}

Index Terms-Wireless sensor network; energy harvesting; battery management; self-sustainable networking; Internet of Things.

\section{INTRODUCTION}

A wireless sensor network (WSN) consists of communicating sensors with the ability to collect, buffer, and transmit information. WSNs are widely used in the context of Internet of Things (IoT). In such a system, interconnected nodes transmit useful measurement information and control instructions via distributed processes [1]. Nowadays, technologies related to the Internet of Things are actively applied, and their advantages are observed in the areas of: logistics, agriculture, marketing, transportation, healthcare, manufacturing [2].

Such IoT tasks are energy demanding, and not all devices can be attached to power outlets. Thus, one can think of providing devices with batteries; but wireless devices can be located in hard-to-reach locations and therefore battery replacement can cause high expenses. Hence, mechanisms to harvest energy from renewable ambient sources (solar,

This work has received funding from the European Unions Horizon 2020 research and innovation program under the Marie Skłodowska-Curie grant agreement No 675891 (SCAVENGE). wind, vibrations etc.) for the battery replenishment are a further development that can significantly enhance the field of applicability of WNSs [3]. However, due to the instability of energy supply from ambient energy sources, it is necessary to develop efficient policies of energy consumption, so as to achieve a satisfactory service rate.

If a battery gets depleted, the wireless device will stop operating and will not be able to transmit data anymore. This situation often occurs when adopting an aggressive strategy of battery usage, i.e., when data packets are transmitted with extremely high service rate [4]. Another situation is observed if packets arrive faster than the device can transmit them (such as in a burst transmission), so the device inserts them into the queue [5]. This could lead to an event where the device data buffer is full and due to the finite maximum queue size, no more data packets could be buffered and there is no other choice rather than to reject excess packets; opposite to the previous event, this happens when the service rate is relatively low. Such situation could be observed in transport layer operations, e.g., due to a badly managed TCP queue. In addition, the more congested the data queue, or in other words, the longer the line of waiting packets to be dispatched, the higher the queueing delay [6]. Based on the afromentioned reasons, the development of an efficient operation strategy should take into account both reduction of data losses and also reduction of the battery inactivity time.

In this paper, we introduce the following contributions. First, we perform simulations of a Markov model designed to keep into account energy harvesting, but also some nonidealities of the battery management. This is done to analyze the evolution of system parameters, and to identify the key variables required to predict possible undesirable events, such as apparent discharges and data losses.

Based on these observations, as a second contribution, we formulate some possible criteria, where tracking just a limited number of parameters, we are able to obtain a sufficiently effective self-control management, where the number of undesirable events is significantly reduced. We believe that this can be a first step in direction of developing policies for selftuning control of energy harvesting WSN. Indeed, as a further evolution of the present paper, we conjecture that autonomic policies can be thought and derived so as to allow efficient management without any prior knowledge on the device itself and/or the energy harvesting and the data arrival processes.

The rest of this paper is organized as following. In Section II, we discuss models of battery and device operation proposed 
in the literature, and battery imperfections considered in previous papers and possible objectives of an efficient operational strategy. In Section III, we give a short description of the double-queue model, its parameters, rules, and conditions. The numerical results of simulations are described in Section IV. Here is proposed the simplified self-management of a battery expressed by restrictions, which are integrated into the model; moreover, we evaluate the efficiency of the proposed management strategy. The paper is concluded in Section V, where possible further developments are outlined that are left for future work.

\section{BACKGROUND}

Many recent papers challenged the task of identifying battery-efficient operation policies for energy harvesting devices in the IoT.

In [7], authors considered a network of nodes exchanging information over a shared channel. In order to optimize the battery work duration (i.e., to reduce battery degradation state), a random channel access scheme is proposed based on an aging-aware Binary Exponential Backoff algorithm, whose objective is to avoid excessive charges and discharges of the battery. A similar idea is used in [8], in which a doublethreshold policy is considered as an optimal strategy; storage and retrieval of energy happens in connection with these thresholds. In particular, the authors developed a water-filling algorithm, which is based on the double-threshold structure. In [9], it is proved that the threshold structure of the policy while using the Markov decision process approach is optimal. In addition, the optimal strategy is formulated with maximization of channel gain as the objective function. A dynamic approach is considered, where energy storage losses are included in the model. The effect of different management policies on battery degradation using a Markov degradation model are also analyzed in [10].

The aforementioned works describe the offline models of battery operation, meaning that model parameters are known, such as energy arrival records, past history, system status. In [17], [11], and [12] the situation of the online optimization of the policy is considered, whenever information about the device is unknown or partially unknown (statistical knowledge of the environment is required). The idea of incomplete available information regarding system parameters for development of the self-control battery management system is something that can be studied and discussed further.

The operational policies can follow different purposes, so the objective function can take different forms:

- minimization of transmitting completion time for a given number of data packets (in offline settings) [5]

- minimization of battery health degradation [7]

- maximization of channel usage [9]

- minimization of energy overflow [8]

- maximization of the battery lifetime [10]

- maximization of the network sum rate [12]

For the development of the optimal policy, different operating battery models are considered. State of the art articles usually focus on battery operation considering different kinds of imperfections and their combinations. As the model gets more realistic, a better operation strategy can be developed. In the literature, the following aspects representing battery imperfections are taken into account:

- battery leakage seen as a decrease of the energy level, due to chemical degradation of the active materials [13]

- imperfect state of charge knowledge expressed as unavailability of the precise level of energy [11]

- charge recovery modeled as an increase of battery level, when no energy is diminished by both leakage or the discharge event due to the data transmission. This effect appears because of non-homogeneous distribution of the electroactive species within a battery, which get equalized because of the chemical diffusion phenomena [4]

- deep discharge characterized by decreasing of the apparent energy level, due to the unbalanced electroactive material distribution [13], which are happening only within service (or data transmission).

- charging/discharging imperfections, meaning that only a limited portion of the available energy can be stored in the battery during charging [8]

We considered the operation policies, which are based on the battery models with imperfections. For example, in [15] authors presented the policy optimization problem, which optimizes the quality of the service and battery lifetime taking into account the degradation of the battery. The linear programming optimization algorithm can be applied for this formulation. In [16] similar optimization problem is formulated for hybrid electric vehicles, based on dynamic programming theory. Authors of [17] showed the effect of real battery constraints on the thoroughput optimization problem and a comparison between the ideal and real model. It was shown that ignoring real battery effects could even lead to zero throughput. The effect of leakage was considered in [18] and the optimization framework was proposed in order to optimize the amount of data transmitted within a given transmission deadline. In [19], it is highlighted that the leakage can lead to $10-20 \%$ of energy loss, thus, throughput optimization was proposed, subject to energy stochastic constraints, and a dynamic-programming type algorithm was offered as well. In this paper, we wanted to avoid similar optimization formulations, which requires lots of computational effort to be solved, instead we devise a simple policy with limited number of requiered parameters. We will take into consideration such imperfections as the battery leakage, deep discharge, and charge recovery. This means that our energy queuing model for the battery involves events where the battery is discharged even when data is not sent (leakage), or it gets discharged more than it should (deep discharge). This leads to an apparent energy level which is lower than the actual one [13], but on the other hand a charge recovery effect may be present, leading the apparent energy level to raise towards the actual one when the battery is not used.

Within this setup, we focus on undesired events such as battery outage, corresponding to depletion of the energy queue, and data overflow, i.e., an excess in the data queue implies that some packets to be discarded. This happens when the 
service rate is too high or too low, respectively. However, the exact definition of "high" or "low" strongly depends on the entire system parameters of data and energy queues, also including non-idealities such as the leakage rate, that are impervious to estimate. Our goal will be to identify which essential parameters can be employed to set rules to regulate the service rate, so as to avoid the aforementioned undesirable events, still keeping the management simple.

\section{MODEL}

We consider a model for an energy harvesting device (wireless transmitter, sensor node), which transmits data packets and is powered by a battery, recharged by a harvesting mechanism. In this work, we use the model proposed and discussed in detail in [13]. In this chapter, we will briefly describe the model parameters, rules and restrictions.

The model is characterized by two buffers for data and energy, i.e., double queue system, with the energy buffer being nothing else than the battery of the device. The formalization of a data queue is self-explanatory, albeit we assume that packets are all identical in size, for the sake of simplicity. Instead, seeing the battery as an energy queue requires the assumption that the energy flow is discretized into energy quanta, with a quantum being the amount of energy for transmitting one data packet. Thus, the energy queue is effected by processes related to generation, storage, and consumption of energy quanta. Similarly, the data queue evolves according to arrival, buffering, and transmission of the data packets. The two events of energy consumption and data transmission take place simultaneously. In addition, to account for nonidealities we actually consider two different values for the energy level. We may consider the value of energy level that is available at the electrodes of the battery (apparent energy level) and the true energy level stored in the battery. Due to degradation phenomena of the battery [10], in case a packet is transmitted, the true energy level decreases by one quantum, but the apparent energy level may decrease by more than that.

We introduce the following assumptions and parameters that regulate the system model and subsequently will be implemented by our simulation [4].

1) Time is slotted (or discretized) with a unit slot length of arbitrary durations. All the metrics are evaluated on a slot-by-slot scale, even though we drop the time index $t$ for notational brevity.

2) Data are transmitted as packets of identical size and whose number in the data queue is denoted by $q$.

3) Data are generated according to an independent homogeneous Bernoulli processes of rate $\lambda$, meaning that every packet arrives to the queue with probability $\lambda$ or does not arrive with probability $1-\lambda$ at any time slot.

4) We consider a finite size for the data queue, so the maximum length of the queue is equal to $Q$.

5) Energy is generated according to an independent homogeneous Bernoulli processes of rate $\eta$, so every quantum of energy arrives to the queue with probability $\eta$ and does not arrive with probability $1-\eta$ at any time slot.

6) Two energy levels are considered in the model: apparent and real denoted by $a$ and $e$ respectively.
7) The incoming energy is stored in a rechargeable battery with finite capacity $E$ (maximum length of the energy queue). Thus, $0 \leq a \leq e \leq E$ holds at all times $t$.

8) The service rate of both queues is denoted as $\mu$. Data packets are also served according to an independent homogeneous Bernoulli process, however, both queues must serve one client at the same time, so as to reflect that energy is spent if there is a data packet to transmit. The value of $\mu$ depends on the operation policy: the higher $\mu$, the more aggressive the battery usage.

9) Leakage is presented by parameter $\gamma$, decreasing the energy level (both apparent and the real one) by 1 within a time slot. Leakage happens only when no service occurs in a time slot.

10) Recovery effect can also happen when $a<e$, and this occurs according to a superimposed independent Bernoulli process, whose rate is $\beta$ if $e \neq E$, and $(1-\gamma)$ if $e=E$.

11) Whenever a package is transmitted, a battery discharge happens. This can either be deep, with probability $\alpha$, or normal, with probability $1-\alpha$.

12) The gap between $a$ and $e$ is denoted as $\Delta$.

The system state is characterized by 3 parameters: number of packets in the data queue, apparent energy level and true energy level $(q, a, e)$. System state changes during time happens by the following rules, where $[x]^{+}=\max (x, 0)$.

Packet arrival: $q \rightarrow \min \{q+1, Q\}$

Energy arrival: $a \rightarrow \min \{a+1, E\}, e \rightarrow \min \{e+1, E\}$

Charge recovery: $a \rightarrow \min \{a+1, E\}$,

Leakage: $a \rightarrow[a-1]^{+}, e \rightarrow[e-1]^{+}$

Normal discharge: $a \rightarrow[a-1]^{+}, e \rightarrow[e-1]^{+}$

Deep discharge: $a \rightarrow \max \{a-2, e-\Delta-1\}, e \rightarrow[e-1]^{+}$ Moreover, we also impose the following restrictions to the system's dynamics.

1) Energy arrivals as well as transmissions, simultaneously take place within the time slot. This means that a packet or a quantum of energy arriving at the time instant $t$ cannot be immediately exploited but are available for transmission or use from time $t+1$.

2) $0 \leq q \leq Q$, the size of the data queue cannot exceed the maximum data buffer size.

3) $0 \leq a \leq e \leq E$, the size of the apparent and true energy level cannot exceed the battery capacity.

4) Discharges can happen only if $a>0$; it is the apparent level, indeed, that dictates whether the node can or cannot transmit data.

5) The gap between $a$ and $e$ cannot be too high. So a deep discharge can only happen if $e-a<\Delta$.

6) Leakage and deep discharge cannot happen in the same time slot, thus $\gamma+\alpha \leq 1$.

7) Leakage and normal discharge cannot happen in the same time slot, thus $\gamma+(1-\alpha) \leq 1$.

8) Leakage and recovery cannot happen in the same time slot, thus $\gamma+\beta \leq 1$.

To evaluate the effectiveness of the proposed conditions, we will consider the battery outage frequency (apparent and full) and data loss event frequency, which happens when at 
TABLE I: Simulation Parameters and Results

\begin{tabular}{|l|c|c|}
\hline Parameters & Low stress & High stress \\
\hline Packet arrival rate $\lambda$ & 0.5 & 0.5 \\
\hline Energy arrival rate $\eta$ & 0.6 & 0.6 \\
\hline Max queue size $Q$ & 10 & 10 \\
\hline Max battery size $E$ & 10 & 10 \\
\hline Deep discharge rate $\alpha$ & 0.4 & 0.5 \\
\hline Charge recovery rate $\beta$ & 0.1 & 0.1 \\
\hline Max gap of apparent/true energy level $\Delta$ & 6 & 6 \\
\hline Leakage rate $\gamma$ & 0.1 & 0.1 \\
\hline Service rate $\mu$ & 0.4 & 0.7 \\
\hline Results & & \\
\hline Apparent discharge freq. & $0.5 \%$ & $3.1 \%$ \\
\hline Data loss event & $2.5 \%$ & $0.0 \%$ \\
\hline
\end{tabular}

time $t$ we observe $q=Q$ and a new packet is to arrive to the data queue in the next time slot. Battery discharge is the energy outage event when $e=0$ and therefore the device is inactive until new energy has arrived. The apparent discharge event happens when $\{a=0 \mid e \neq 0\}$, while full discharge event happens when $\{a=0 \mid e=0\}$. Note that in the apparent discharge event, the node stops transmitting but can restore its operation simply when the energy levels get equalized by the charge recovery effect.

\section{Simulation And Numerical Results}

In this section, we manage the simulations to analyze the behavior of model parameters and possibility to reduce the number of variables needed to predict possible negative events. For this purpose, we will analyze the frequency of apparent and full discharge events. We consider different values of the service rate $\mu$, specifically leading to: a Low stress situation for the battery, where $\mu<\eta$, and a High stress case, where $\mu>\eta$, which we expect may cause several apparent discharges in the battery. This serves to see how the service rate influences the frequency of the apparent discharge event. Specifically, the parameters are chosen as $\eta=0.5$ and thus $\mu=0.4$ (for Low stress) and $\mu=0.7$ (for High stress). Table I reports the simulation parameters and also summarizes the most relevant results. For the following instance, data was generated randomly with the respect to the parameters in Table I and time horizon $t=1000$.

Most notably, Table I reports that for the battery under low stress $(\mu=0.4)$, the event of data loss is more frequent than the apparent discharge. We observe the opposite situation if the battery is highly stressed, i.e, for $\mu=0.7$, in which the data loss event does not happen at all.

We better analyze the results by showing the full evolutions of two sample simulations in Figs. 1-2, showing the cases of low and high battery stress, respectively. In the former case, it is visible from Fig. 1 that a lower service rate leads to a higher probability of data loss event. Conversely, Fig. 2 shows how higher service rate leads to apparent outages and minimization of the data loss events. Furthermore, we can plot the occurrences of these two events in a more generalized setup, where $\mu$ is varied from 0.1 to 1 ; the result is reported in Fig. 3, which confirms what observed before. Thus, we can identify that there is a connection between the data buffer occupancy (i.e., the $q / Q$ ratio) and the apparent discharge and data loss events.

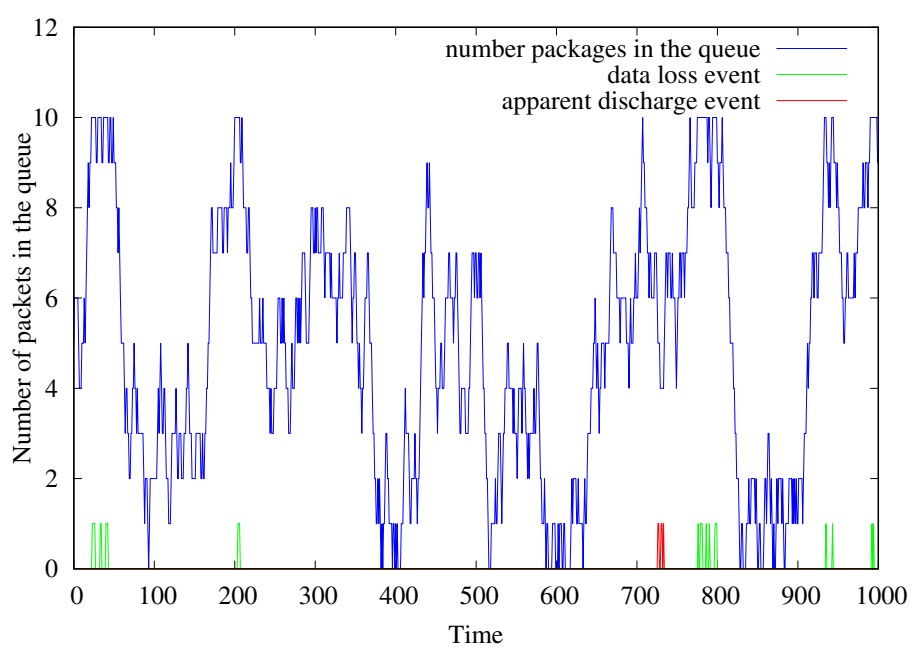

Fig. 1: Apparent dischargeand data loss event for $\mu=0.4$

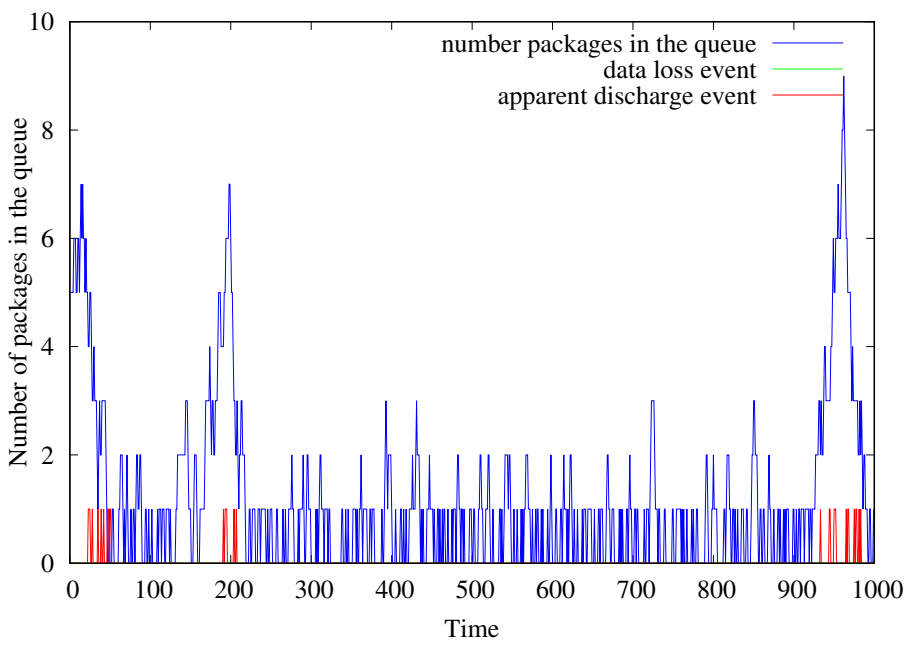

Fig. 2: Apparent discharge and data loss event for $\mu=0.7$

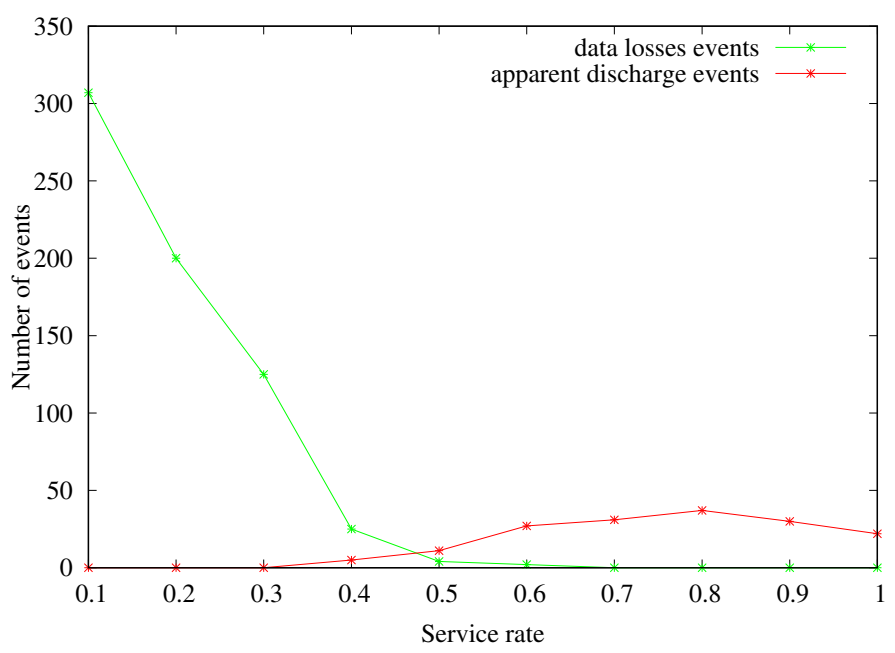

Fig. 3: Changes of data loss and apparent discharge frequency for $\mu=0.1-1.0$. 


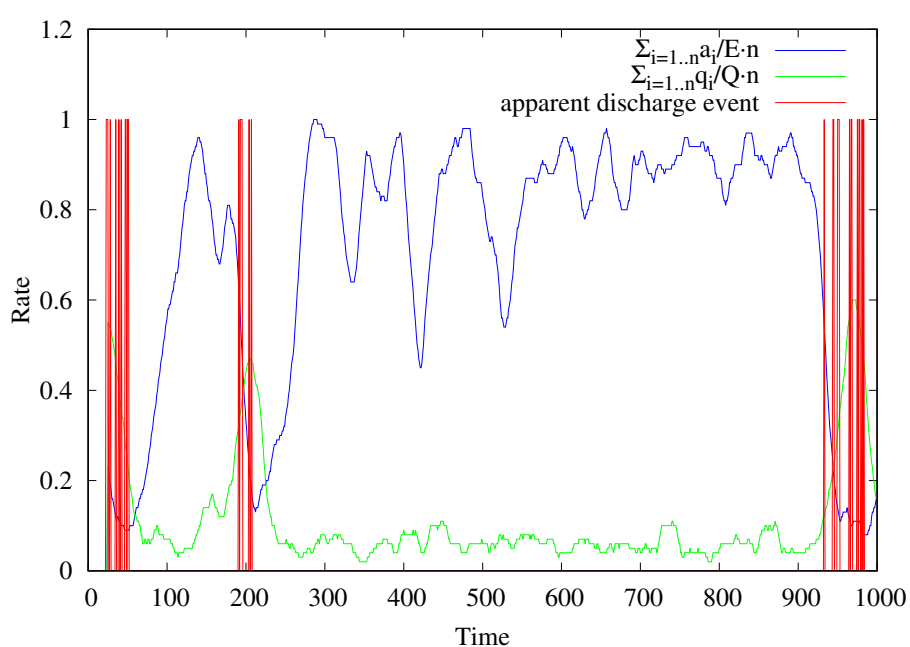

Fig. 4: Comparison of condition (1) with apparent discharge events, for $n=25$, in the High stress scenario $(\mu=0.7)$.

Now, considering a scenario where $\mu$ can be regulated by the device, and recalling that $q=Q$ is the condition triggering a data loss event, it is clear that keeping $q / Q$ as relatively close to 1 may increase the frequency of data losses. However, increasing the rate so as to keep $q / Q$ low also incurs a likely situation where apparent battery outages appear. More in general, the relationship between $\mu$ and $q / Q$ may be descriptive of this tradeoff. On one hand, the value of $q / Q$ has to be minimized, which is possible by increasing the value of service rate $\mu$. On the other hand, high values for $\mu$ is responsible for a fast battery discharging and increasing of apparent/full discharge probability. So the aim is to find optimal relationship between $q / Q$ and $\mu$.

However, we remark that $q / Q$ can change in a time slot when packets leave the data queue, which would imply an energy consumption, but also when packets arrive, that has nothing to do with the energy level. Conversely, the energy level changes also because of deep discharges, leakage, or charge recovery effects, and none of these phenomenas is reflected by a change of $q$. Thus, the current value of $q / Q$ cannot exactly predict that the apparent/full discharge event is about to happen, also the previous states of the battery needs to be taken into account. For this reason, instead of just the local value of $q$ and $a$, we propose to consider a moving average value of $q / Q$ and $a / E$ for $n$ steps. Therefore, we seek to establish a condition that takes form:

$$
\sum_{i=1}^{n} \frac{q}{n Q}-\sum_{i=1}^{n} \frac{a}{n E}<0
$$

This condition can be better observed in Fig. 4, where we plot, for an instance of the system under the High stress case $(\mu=0.7)$ the time evolution of the two terms involved in (1) and we also highlight, in red, the occurrence of the apparent discharge event. For this specific evaluation, we chose $n=25$. As we can see from Fig. 4, apparent discharge events occur only when (1) is violated.

To describe the data loss, we obviously involve again the value of $q / Q$, but we want to derive a connection to the battery

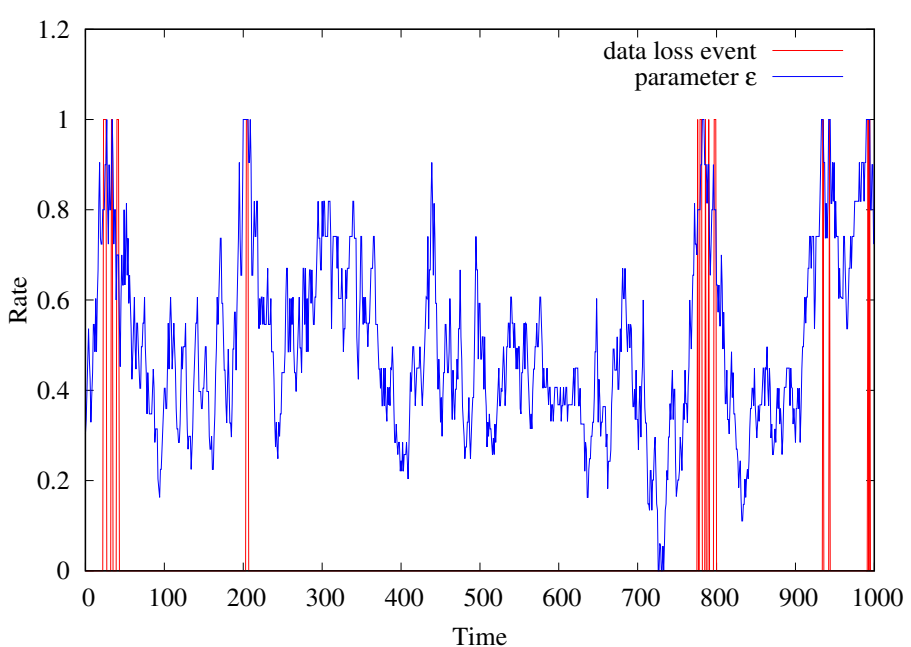

Fig. 5: Data loss parameter values $(\mu=0.4)$

level as well. We denote with $\epsilon$ the data loss probability. From empirical observations, we found out that the following relationship is often closely achieved, which establishes an exponential behavior of the data loss probability versus the ratio $q / Q$ but also linear in the apparent energy level:

$$
\epsilon=\frac{a}{E \cdot e^{1-\frac{q}{Q}}}
$$

The initial assumption is that the probability of data packet transmitting is equal to the relative energy level of the battery. But while our goal is not to let the buffer to get full $(q=Q)$, we adjust $\epsilon$ by $1 / e^{1-\frac{q}{Q}}$. Higher value of $q / Q$, performs less correction (decrease) of $\epsilon$.

Let us consider changing of the $\epsilon$ value for the case of Low Stress of the battery $(\mu=0.4)$. In this case, $\epsilon$ takes the maximum value of 1 exactly wherever there are data loss events. This is plotted in Fig. 5, where data loss events are highlighted in red.

Now, we extend these conditions to an active control of the data packet transmissions. In particular, we employ these remarks about the aforementioned connections to establish some preliminary checks in the decisions to be made by the wireless device on whether to transmit a packet or not. We add two more conditions to the model, reflecting (1) and (2), that is, a packet can be transmitted only if $(q / Q)-(a / E)<0$ (condition 1) and $a\left(E \cdot e^{1-\frac{q}{Q}}\right)^{-1}<1$ (condition 2). If both conditions are met, then we allow data packets to be sent (the actual decision on whether to transmit a packet is made depending on the service rate and the state of the system). If condition 1 is violated (that is, the energy level is estimated to be low) and condition 2 is not, then the data packet is held and cannot be transmitted. If the opposite happens, i.e., the energy level is sufficient but conversely there is an abundance of data in the queue and the buffer risks an overflow, we force packet transmission, i.e., we transmit with service rate 1 . Note that this heuristic rule is anyway not almighty, since if both conditions are violated, it means that we estimate that both energy outage and data overflow are about to occur, but there is 


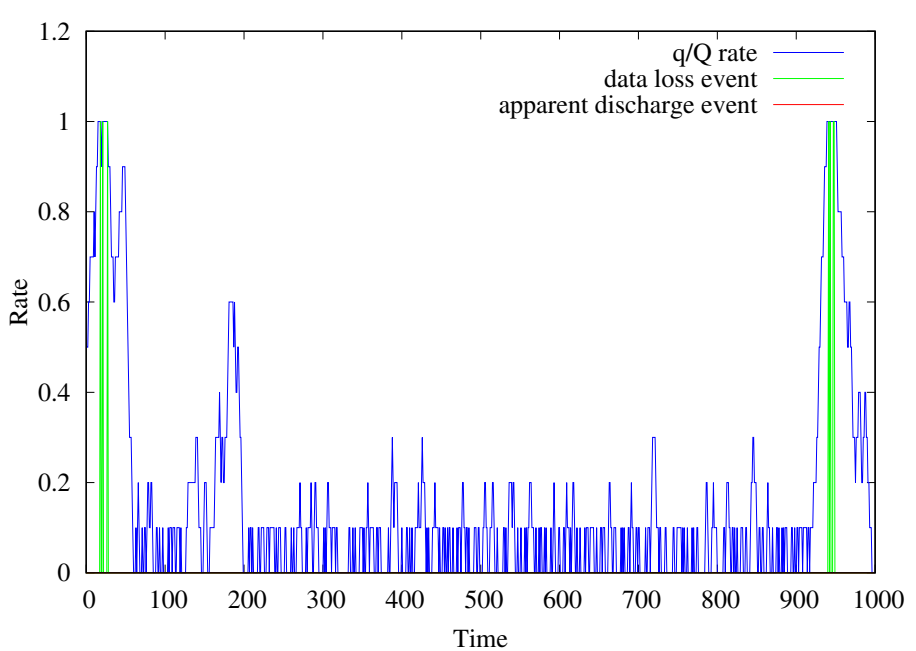

Fig. 6: Apparent discharge event $(\mu=0.7)$

nothing that can be done, since any data sent to avoid overflow will probably be lost because of low battery.

To examine conditions (1) and (2) we integrated these inequalities into the simulation models for the example considered above. The results of the simulations are presented on Fig. 6, where we consider a case of High stress for the battery $(\mu=0.7)$. The figure shows that integrating restrictions (1) and (2) reduces the number of apparent discharge events. For the previous example, when $\mu=0.7$, integrating these conditions reduces the number of apparent discharge events by $77.4 \%$. Also, we verified that, similarly to this case, when $\mu=0.8$ we have a reduction of $75.6 \%$ of the apparent outage events, when $\mu=0.9$ this reduction is of $73.1 \%$, which means that this result is pretty consistent also for other values of $\mu$.

\section{Conclusions}

We have demonstrated the effect of battery operation policy and evaluated the occurrence of undesired events which could cause negative consequences, for example, battery inactivity. We based on a model which deals with the data queue and the energy queue of the device as well as battery imperfections such as leakage, charge recovery and deep discharge. By inspecting the simulation results we found that certain parameters are significant for prediction of such events as apparent discharge and data loss. Based on these parameters, we proposed a simplified self-control managment for a battery, which is to verify the conditions in the model decision making process for battery-powered energy harvesting mobile devices. The purpose of the self-control management is to reduce both the data losses that happen when the data buffer is full, and the apparent outage of the device. The restrictions integrated in the strategy can be easily rewritten to take into account full outage of the device. The effectiveness of the proposed scheme was numerically proved.

For future work, it is possible to consider the situation where some information is unknown or hidden (imperfect state of charge knowledge). Another possibility for the future work is to generalize the regulation of the $\mu$ parameter of self-turning control systems (STC). STC systems are based on the derivation of the parameters to adapt and achieve an optimal state of a change of parameters in situation of unsteady external conditions, which is possible to realize by parameter value estimation based on recorded statistical information.

These considerations may shed new light on the possibility to design a fully autonomic sensor device that is not only capable of energy harvesting, but also to exploit it at its fullest to reach a true energy independence, which would be key to guarantee successful and durable IoT applications.

\section{REFERENCES}

[1] L. Atzori, A. Iera, and G. Morabito, "The internet of things: A survey," Comp. Netw., vol. 54, no. 15, pp. 2787-2805, 2010.

[2] L. Da Xu, W. He, and S. Li, "Internet of things in industries: A survey," IEEE Trans. Indust. Inf., vol. 10, no. 4, pp. 2233-2243, 2014.

[3] A. Sinha and A. Chandrakasan, "Dynamic power management in wireless sensor networks," IEEE Design and Test of Computers, vol. 18, no. 2, pp. 62-74, 2001.

[4] N. Michelusi, L. Badia, and M. Zorzi, "Optimal transmission policies for energy harvesting devices with limited state-of-charge knowledge," IEEE Trans. Commun., vol. 62, no. 11, pp. 3969-3982, 2014.

[5] J. Yang and S. Ulukus, Optimal packet scheduling in an energy harvesting communication system, IEEE Trans. Commun., vol. 60, no. 1, pp. 220-230, 2012.

[6] K. W. Ross, J. F. Kurose, Computer networking: a top-down approach. "Delay and loss in packet-switched networks". chapter 1.4, Addison Wesley, 2007.

[7] R. Valentini, M. Levorato and F. Santucci, "Aging aware random channel access for battery-powered wireless networks", IEEE Wirel. Comm. Lett., vol. 5, no. 2, pp. 176-179, 2016.

[8] K.Tutuncuoglu, A.Yener, S. Ulukus, "Optimum policies for an energy harvesting transmitter under energy storage losses", IEEE J. Sel. Ar. Commun., vol. 33, no. 3, pp. 467-481, 2015.

[9] A. Biason and M. Zorzi, Energy harvesting communication system with SOC-dependent energy storage losses, Proc. ISWCS, pp. 406410, 2015.

[10] N. Michelusi, L. Badia, R. Carli, L. Corradini, and M. Zorzi, "Energy management policies for harvesting-based wireless sensor devices with battery degradation," IEEE Trans. Commun., vol. 61, no. 12, pp. 49344947, 2013.

[11] D. Del Testa, N. Michelusi, and M. Zorzi, "Optimal transmission policies for two-user energy harvesting device networks with limited state-ofcharge knowledge" IEEE Trans. Wirel. Commun., vol. 15, no. 2, pp. 1393-1405, 2016.

[12] R.-S. Liu, P. Sinha, and C. Koksal, "Joint energy management and resource allocation in rechargeable sensor networks", Proc. IEEE Infocom, pp. 1-9, 2010.

[13] L. Badia, E. Feltre, and E. Gindullina, "A Markov model accounting for charge recovery in energy harvesting devices," accepted in IEEE WCNC Workshop on Energy Harvesting and Remotely Powered Wireless Communication for the IoT, 2017

[14] U. Boker, T. Henzinger and A. Radhakrishna, "Battery transition systems," Proc. ACM SIGPLAN-SIGACT POPL, 2014.

[15] N. Michelusi, L. Badia, R.Carli, L. Corradini and M. Zorzi "Impact of battery degradation on optimal management policies of harvestingbased wireless sensor devices", Proc. IEEE INFOCOM, Turin, Italy, pp. 590-594, 2013.

[16] F. Martel, S. Kelouwani, Y. Dube and K. Agbossou, "Optimal economybased battery degradation management dynamics for fuel-cell plug-in hybrid electric vehicles" J. Power Sources, vol. 274, pp. 367-381, 2015.

[17] A. Biason and M. Zorzi, "On the effects of battery imperfections in an energy harvesting device", Proc. IEEE International Conference on Computing, Networking and Communications, pp. 942-948, 2016.

[18] B. Devillers and D. Gunduz "A general framework for the optimization of energy harvesting communication systems with battery imperfections", journal of communocations and networks, vol. 14, no. 2, pp. 130-139, 2012.

[19] F. Yuan, Q.T. Zhang, S. Jin, and H. Zhu "Optimal Harvest-Use-Store Strategy for Energy Harvesting Wireless Systems", IEEE transactions on wireless communications, vol. 14, no. 2, pp. 698-710, 2015. 